\title{
The effects of chronic alcohol exposure on gene expression of calcium handling proteins in skeletal muscle
}

\author{
T. Nakahara ${ }^{1}$, K. Hashimoto ${ }^{1}$, M. Hirano ${ }^{1}$, R. Rajendram ${ }^{2}$, J. Harris ${ }^{2}$, K. Ohlendieck ${ }^{3}$ \\ and V. R. Preedy ${ }^{2}$ \\ ${ }^{1}$ National Hospital Organization, Hizen Psychiatric Center, Kanzaki, Saga 842-0192, Japan, ${ }^{2}$ Nutritional Sciences Division, \\ King's College London, London SE1 9NU, UK and ${ }^{3}$ Department of Biology, National University of Ireland, Maynooth, \\ Co. Kildare, Ireland
}

\begin{abstract}
Alcoholic myopathy is a common pathology affecting 40-60\% of all chronic alcohol misusers. The myopathy is characterised by muscle weakness and loss of protein content. Women are particularly vulnerable, but the mechanisms for either the myopathy or the differential gender-based sensitivities are unknown. We hypothesised that alcohol-induced defects in muscle are due to perturbations in calcium channel proteins, particularly at the level of gene expression. To test this we used a well-validated rat model of alcohol exposure, and then measured mRNA levels by quantitative RT-PCR. Thus, male or female Wistar rats were fed nutritionally complete liquid diets containing ethanol as $35 \%$ of total dietary energy. Controls were pair-fed identical amounts of the same diet in which ethanol was replaced by isocaloric glucose. Pair feeding thus ensured that apart from energy derived from ethanol or glucose, the intake of all micro- and macronutrients were identical in both control and treated rats for a particular gender. At the end of 6 weeks, the rats were killed and skeletal muscles, as represented by the gastrocnemius, were dissected. The relative abundance of mRNAs encoding triadin, the alpha- 1 dihydropyridine receptor and calreticulin were then measured by quantitative RT-PCR. Data were analysed by two-way ANOVA.
\end{abstract}

Table 1. Effects of chronic ethanol feeding on gene expression in rat skeletal muscle. The relative abundance of mRNAs were quantified by RT-PCR relative to GAPDH. Data are expressed as mean densitometric units. There were $n=10$ (male) or $n=8$ (females) rats in each group

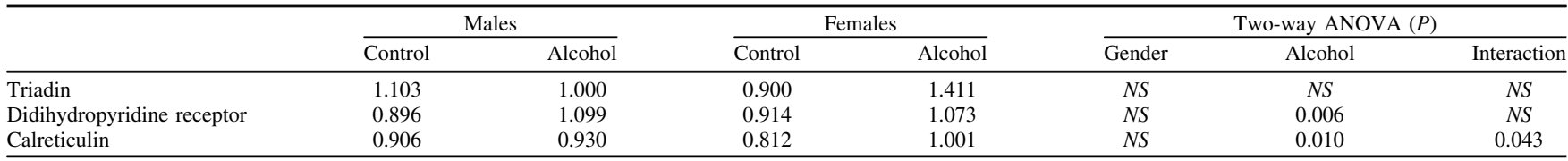

The data (Table 1) showed significant effects of alcohol on the mRNAs encoding calcium handling proteins and some evidence for differential sensitivities. This has particular relevance for skeletal muscle as calcium regulation is necessary for functional activity and weakness is a distinguishing feature of alcohol toxicity. For example, the transverse tubules in muscle contain the multimeric dihydropyridine receptor that has five subunits, one of which (i.e., the alpha 1) was investigated in this study. Calreticulin is one of the $\mathrm{Ca}^{2+}$ storage proteins and is involved in the fine regulation of the ryanodine receptor. Up-regulation of both the alpha-1 dihydropyridine receptor and calreticulin genes may represent an adaptive mechanism although further analysis is being carried out to explore other genes involved in calcium regulation. 